\title{
INFORME 2012 DE LA RIED. REVISTA IBEROAMERICANA DE EDUCACIÓN A DISTANCIA
}

Es preceptivo que cada año la dirección de la RIED, Revista Iberoamericana de Educación a Distancia, elabore un informe que ha de ser presentado al Consejo Asesor, que coincide con el Consejo Directivo de la Asociación Iberoamericana de Educación Superior a Distancia (AIESAD) que es el órgano de quien depende la RIED.

Durante los últimos años se ha trabajado incesantemente para que la RIED cumpla con todos los criterios de calidad que exige la actual situación de las revistas científicas. Las memorias de actividades de la RIED de los años anteriores son un fiel reflejo de la madurez que ha alcanzado como revista científica de calidad. Las medidas y criterios, establecidos en épocas pasadas, comenzaron a dar sus frutos. En estos momentos la RIED, Revista Iberoamericana de Educación a Distancia, cumple con todos los indicadores propuestos tanto por la Fundación Española para la Ciencia y la Tecnología (FECYT), como por las agencias de calidad y otras bases de datos nacionales e internacionales.

No obstante, en el informe anterior correspondiente al año 2010-2011, se trató de una serie de propuestas de mejora que se consideraba recomendable implementar dentro de nuestra revista para subsanar algunas carencias encontradas, y por ende, mejorar la propia calidad científica de la revista. Los aspectos de mejora que se nombraban eran ejes transversales dentro de los indicadores de calidad, por lo que se presentan en este documento como aspectos de mejora que son desarrollados en cada uno de los criterios de calidad que debemos atender.

No obstante, seguimos, como es lógico, atendiendo las indicaciones de las agencias de calidad, y para ello debemos centrarnos en unos aspectos destacados que a continuación profundizaremos:

- Criterios de calidad informativa de la revista como medio de comunicación científica. 
- Criterios sobre la calidad del proceso editorial.

- Líneas futuras. Criterios sobre la calidad científica de la revista.

\section{CRITERIOS DE CALIDAD INFORMATIVA DE LA REVISTA COMO MEDIO DE COMUNICACIÓN CIENTÍFICA}

Son muchos los criterios de calidad informativa que se tienen en cuenta como medio de difusión. La RIED, Revista Iberoamericana de Educación a Distancia, cumple con la mayoría de ellos. Por esta razón, en este apartado no nos vamos a detener en todos, sino que es importante centrarnos en aquellos que han sido un objetivo primordial para alcanzar las cuotas de calidad más altas. De esta forma, este apartado se centrará en tres aspectos relevantes:

- La apertura exterior del Comité Científico y los Evaluadores Externos.

- El cambio de licencia de Copyright a Creative Commons.

- El contador de visitas de la Web como medio de difusión mediática.

- Intercambio y suscripciones.

- OJS para el aumento del impacto mediático.

- Indización. Inclusión en nuevas Bases de datos.

\section{Apertura exterior del Comité Científico y los Evaluadores Externos}

Sin duda, uno de los logros más relevantes en la RIED durante su evolución es la conformación de su Comité Científico y Evaluadores Externos. En el momento actual nuestra revista cuenta en este capítulo con una red de expertos en el área de la educación a distancia de reconocido prestigio internacional, y conformado por 45 doctores/as de diversas nacionalidades provenientes de distintos centros de investigación y universidades.

Todos los miembros de la red de expertos (Comité Científico y Evaluadores Externos) son ajenos al Consejo Asesor y su función se centra en asesorar y evaluar la publicación, avalándola científicamente y proyectándola internacionalmente. 
La apertura exterior, tanto de los Evaluadores Externos, como de los miembros del Comité Científico, es un elemento que se tiene en cuenta, y de esta forma, la RIED cumple con uno de los criterios básicos de calidad que solicitan las agencias nacionales e internacionales. Precisamente la apertura de los evaluadores externos es uno de los puntos principales sobre los que se ha trabajado este año. Esta era una de las carencias detectadas en informes anteriores, y por ende, era un elemento primordial en la mejora de la revista RIED. Como se decía en memorias anteriores, y se puede ver en la gráfica siguiente, un buen número de los evaluadores externos y de los miembros del Comité Científico son de nacionalidad española. Sin embargo, queremos resaltar que al tratarse de una revista iberoamericana, necesitamos diversificar el número de los miembros para incluir a más doctores de otras nacionalidades, cumpliendo así con el criterio de apertura al exterior internacional. Por esta razón, este año hemos ampliado la base de los evaluadores externos. Actualmente recibimos multitud de artículos en lengua portuguesa, y como se puede apreciar en el siguiente apartado estamos en la base de datos brasileña CAPES con la calificación $\mathrm{A}++$, la más alta posible. Al recibir ese número tan elevado de artículos en este idioma, hemos incorporado como evaluadores externos este año a 4 doctores brasileños más del área de la educación a distancia que suplan las carencias que existían en el manejo de este idioma. También se incorporó otro experto argentino.

En la siguiente gráfica podemos ver la representación, tanto de los miembros del Comité Científico como de los Evaluadores Externos, de los países iberoamericanos que representan:

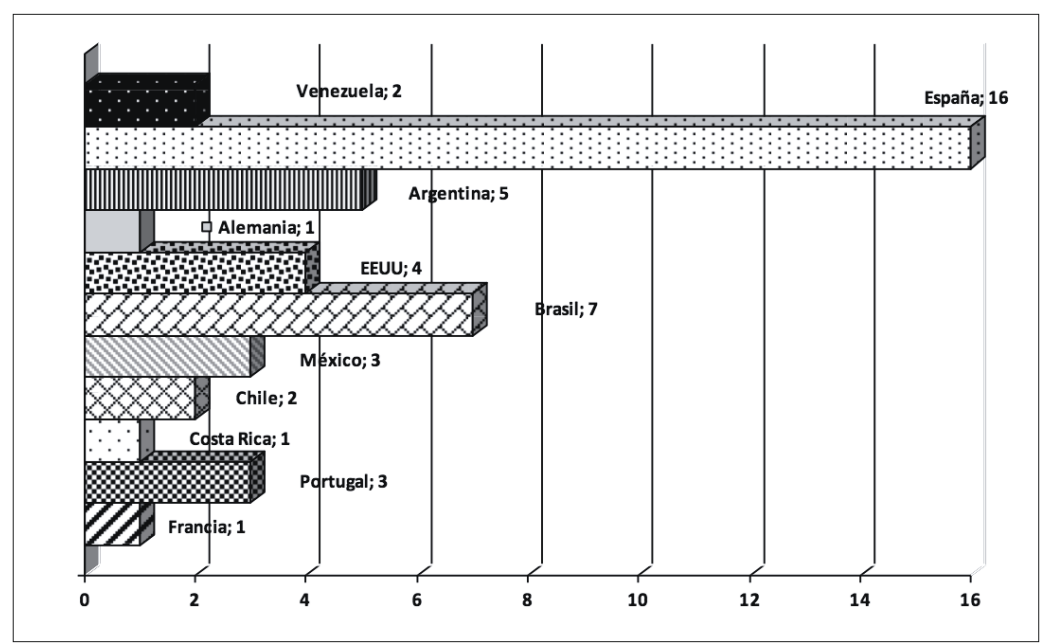

Gráfico 1. Apertura exterior de los evaluadores externos y del Comité Científico 
Como se indicaba anteriormente, a pesar de estas incorporaciones, como se puede observar en el gráfico, la mayoría de los evaluadores externos son españoles, por lo que en estos momentos estamos tratando de ampliar esta red con el fin de diversificar las áreas de conocimiento dentro de este campo y de la diversidad regional.

\section{Cambio de licencia de Copyright a Creative Commons}

Durante el año 2012, la RIED ha apostado también por cambiar la política de licenciamiento de la totalidad de sus contenidos, y de esta manera, aumentar su impacto mediático. La mayoría de las revistas científicas están llevando a cabo igualmente este proceso de cambio con el fin de superar las restricciones e impedimentos derivadas de la clásica licencia de Copyright. Ejemplo de ello son las prestigiosas revistas del Consejo Superior de Investigaciones Científicas (CSIC) de España, cuyos contenidos se han ido acogiendo progresivamente a alguna de las licencias Creative Commons disponibles de forma gratuita.

Las licencias Creative Commnons están inspiradas en la licencia GPL (General Public License) de la Free Software Foundation, y tienen como objetivo posibilitar un modelo legal ayudado por herramientas informáticas para facilitar la libre distribución y el uso de los contenidos científicos. Por este motivo, teniendo en cuenta las ventajas y beneficios que este tipo de licencias podían aportar tanto a los autores como a la difusión de la propia RIED; en el mes de marzo se realizaron una serie de consultas al responsable de Creative Commons España y a los servicios especializados de la UNED con el fin de conocer más al detalle las características y los diferentes tipos de licencias Creative Commons y plantear la posibilidad de acogernos a alguna de ellas.

Finalmente, desde el volumen $\mathbf{1 5 . 2}$ de la RIED, publicado en julio de 2012, nuestra revista queda acogida a una Licencia Creative Commons "ReconocimientoNo comercial 3.O"; lo que significa que los contenidos de la RIED podrán usarse y difundirse libremente siempre que se reconozca la autoría y mientras no se haga un uso comercial de los mismos. El texto y el logo que acompaña a la licencia escogida son los siguientes: "Los textos publicados en esta revista están sujetos a una licencia "Reconocimiento-No comercial 3.o" de Creative Commons. Puede copiarlos, distribuirlos, comunicarlos públicamente, siempre que reconozca los créditos de la obra (autor, nombre de la revista, instituciones editoras) de la manera especificada en la revista". 
Al cambiar del Copyright al Creative Commons se han tenido que implementar una serie de acciones. Lo primero cambiar las normas de publicación de la propia revista, en segundo lugar, informar a todos los autores mediante un correo electrónico, y en tercer lugar, divulgar este cambio a través de la Web de la revista para que todas las personas puedan conocer las nuevas normas de publicación antes de enviar un artículo o bien utilizarlo para sus investigaciones.

Y de ahí que el símbolo que ya aparece en la Web de la RIED (http://www.utpl. edu.ec/ried) sea este:

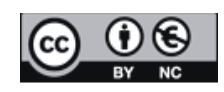

\section{Contador de visitas de la Web como medio de difusión mediática}

Uno de los indicadores que mide la calidad informativa y de difusión de la revista RIED es el contador de visitas situado en su página Web. A través de él podemos comprobar su evolución, el impacto mediático, y el interés que despierta en la Red. La siguiente gráfica 2 contiene las visitas que ha recibido a partir de los datos arrojados por informes anteriores.

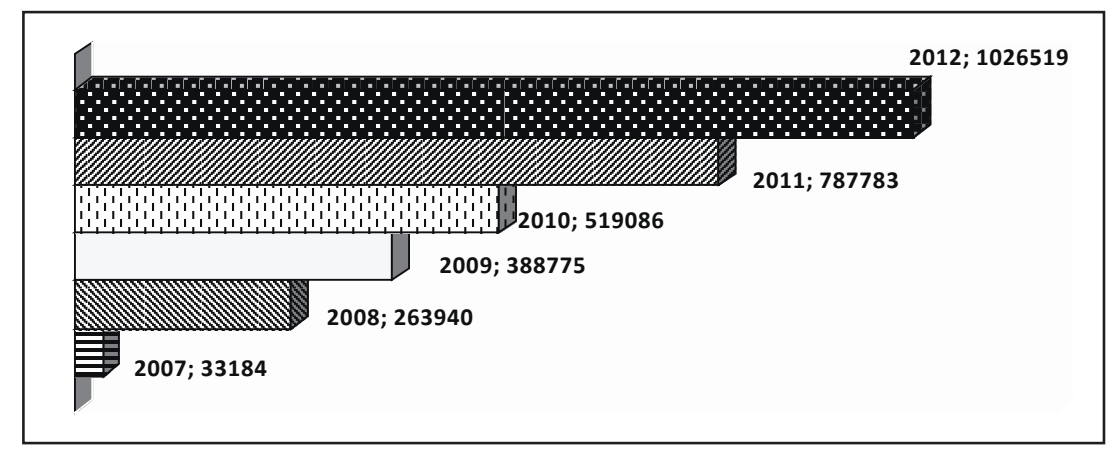

Gráfico 2. Aumento de las visitas recibidas en la página Web de la RIED

A través de estos datos se muestra la evolución y el impacto mediático que ha tenido la página Web. Se puede observar el interés mostrado en la RIED. El aumento de visitas a la revista ha sido muy significativo y de progresión acelerada. 


\section{Intercambio y suscripciones}

Otra de las líneas que marcan los criterios de calidad, en cuanto al impacto mediático de la revista, es el apartado de suscripciones e intercambios. Es necesario establecer unos vínculos de intercambios con otras revistas con el fin de ser introducidos en el mayor número de instituciones posibles, por esta razón, este es otro de los objetivos prioritarios sobre los que este año se ha trabajado.

\begin{tabular}{|l|c|}
\hline $\begin{array}{c}\text { País de origen de la revista con la } \\
\text { que se produce el intercambio }\end{array}$ & $\begin{array}{c}\text { Número de revistas } \\
\text { por país }\end{array}$ \\
\hline Argentina & 5 \\
\hline Brasil & 15 \\
\hline Colombia & 5 \\
\hline Costa Rica & 2 \\
\hline México & 10 \\
\hline Perú & 1 \\
\hline Puerto Rico & 1 \\
\hline República Dominicana & 1 \\
\hline Uruguay & 1 \\
\hline Venezuela & 6 \\
\hline España & 15 \\
\hline Cuba & 1 \\
\hline Ecuador & 3 \\
\hline USA & 1 \\
\hline Chile & $\mathbf{6 9}$ \\
\hline Portugal & 1 \\
\hline Total & 15 \\
\hline
\end{tabular}

\section{OJS para el aumento del impacto mediático}

OJS (Open Journal System) es una herramienta que permite la publicación de los contenidos en abierto, pudiendo ser utilizado únicamente como portal para la difusión de contenidos online. En este sentido, se trata de una herramienta cuya flexibilidad y versatilidad le permite adaptarse a las necesidades y requerimientos de 
cada revista. Por lo tanto, los gestores de cada revista pueden configurar su espacio en $O J S$ para ser utilizado como portal de publicación y/o como herramienta de administración.

En este año 2012 la RIED se ha embarcado dentro de este sistema como medio de difusión y también como medio de gestión. La parte de gestión editorial se verá en el siguiente apartado donde se indicarán los beneficios que se obtienen con esta herramienta, pero no hemos querido dejar pasar la oportunidad que nos ofrece también como medio de impacto mediático.

Una de las ventajas que nos ofrece este sistema es poder mantener dos páginas Web con la información de la revista desde dos sitios diferentes, lo que aumentará nuestra visibilidad. Además, este sistema, al estar dentro de una plataforma de revistas científicas nos abrirá las puertas para obtener nuevos articulistas interesados en publicar en revistas científicas, lo que probablemente aumentará el índice de citas de los artículos.

Por otra parte, una característica muy destacable de OJS consiste en la posibilidad de asignar de manera automática el número DOI a todos los artículos publicados. Recordemos que el DOI (Digital Object Identifier) es un sistema que permite identificar cualquier objeto digital en el ciberespacio con independencia de la URL de la que dependa o del servidor que lo soporte. Por este motivo, el DOI se está convirtiendo en uno de los elementos de calidad a tener en cuenta en cualquier publicación online de carácter científico.

\section{Indización. Inclusión en nuevas Bases de datos}

En el mes de diciembre de 2011 la RIED estaba indizada en:

Bases de datos: DIALNET; DOAJ (Directory of Open Access Journals); GOOGLE Académico; HEDBIB (International Bibliographic Database on Higher Education); IRESIE (Índice de Revistas de Educación Superior e Investigación Educativa); ISOC-Educación (Bases de datos Bibliográficas del CSIC); LATINDEX; MIAR (Matriu d'Informació per a l'Avaluació de Revistes); REDALYC (Red de Revistas Científicas de América Latina, Caribe, España y Portugal).

Plataformas de evaluación de revistas: DICE (Difusión y Calidad Editorial de Revistas); IN-RECS (Índice de Impacto de Revistas Españolas de Ciencias 
Sociales); MIAR (Matriz para Evaluación de Revistas); RESH (Revistas Españolas de Ciencias Sociales).

Directorios selectivos: LATINDEX (Publicaciones Científicas Seriadas de América, España y Portugal); ULRICH’S Periodicals (CSA).

Portales y repositorios especializados: EDUC. AR (El portal educativo del estado argentino); E-SPACIO-UNED (Repositorio institucional de la UNED); $360^{\circ}$ (Plataforma de revistas); UNIVERSIA.

Buscadores de literatura científica: DOAJ (Directory of Open Access Journals); GOOGLE ACADÉMICO; RECOLECTA (Recolector de ciencia abierta); SCIRIUS (For scientific information only).

Catálogos de bibliotecas: BIBLIOTECA DE LA UNAM; BRITISH LIBRARY; BUZ; CARHUS Plus+; Catálogo de la Biblioteca de Educación (Ministerio de Educación, Cultura y Deporte); CCPP (Catálogo Colectivo de Publicaciones Periódicas Español); CIRBIC (Catálogo del CSIC); CCUC; CENDOC; CIDE; CISNE; COMPLUDOC; ICDL; INRP; IOE (Institute of Education. University of London); KINGS; MIGUEL DE CERVANTES; REBIUN; UBACAT; UIB; WORDLCAT; ZDB.

Durante el año 2012 hemos conseguido, además, incluir la RIED en diferentes bases de datos, portales, buscadores y catálogos de Alemania, Brasil, España, Chile, Uruguay, México, Argentina, Colombia, Costa Rica y Reino Unido. A continuación detallamos dichos nuevos índices:

\section{BASES DE DATOS}

BASE. Base de datos de la Biblioteca de la Universidad de Bielefeld. (Alemania). http://goo.gl/IDe2h

Bielefeld Academic Search Engine

1. Revista Iberoamericana de Educación a Distancia (RIED)

Título: $\quad$ Revista Iberoamericana de Educación a Distancia (RIED)

Materias: $\quad$ distance education ; e-learning ; DoajSubjectTerm: Education ; LCC: L7-991 ; LCC: LA5-2396 ; LCC: LB5-3640 ;

Editorial: $\quad$ LCC: LC8-6691 $\quad$ Asociación Iberoamericana de Educación Superior a Distancia

Fecha de publicación: 1998

Lenguaje: Spanish

URL: http://www.utpl.edu.ec/ried/

Proveedor de datos: Directory of Open Access Journals: DOAJ Journals

8. Buscar en Google Scholar $\square$ Enviar este por correo $\square$ Exportar registro $v$ (†) Agregar a favoritos 
CAPES. Coordenação de Aperfeiçoamento de Pessoal de Nível Superior. (Brasil). http://goo.gl/QkY1B

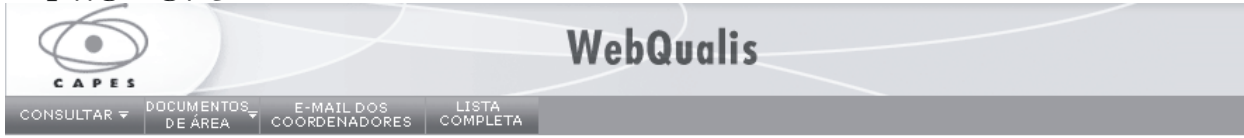

Selecione o tipo de detalhamento da pesquisa:

\begin{tabular}{l|l|l} 
Por ISSN do Periódico Por Titulo do Periódico Por Classificação / Área de Avaliação & P
\end{tabular}

$1138-2783$

PSICODOC. Base de datos de Psicología. (España).

http://www.psicodoc.org/

OSICOdOC

Buscador de Revistas incluidas en PSICODOC

Revistas incluidas en PSICODOC
stas por palabras contenidas en el tiftulo o editor o el ISSN de la revista

\section{Buscar Limpiar \\ Registros... 1 Criterios de búsqueda: RIED \\ ISSN: $1390-3306$ \\ Titulo: RIED Revista lberoamericana de Educación a Distancia [versión electrónica] \\ Editor: Asociación Iberoamericana de Educación Superior a Distancia \\ Direccion: RIED - UTPL. San Cayetano Alto, s/n Loja (Ecuador) \\ URL:http://www.utpl.edu.ec/ried/index.php?option=com content\&task=view\&id=5\&ltemid=6} Email:ried@edu.uned.es

\section{PORTALES Y REPOSITORIOS ESPECIALIZADOS}

\section{Actualidad Iberoamericana. (Chile). \\ http://goo.gl/7dSmC}

\section{Revistas indizadas en Actualidad Iberoamericana}

\begin{tabular}{|c|c|c|c|c|c|}
\hline & & $\begin{array}{l}\text { RIED. Revista } \\
\text { Iberoamericana de } \\
\text { Educaciôn a } \\
\text { Distancia. }\end{array}$ & $1138-2783$ & $\begin{array}{l}\text { Cátedra UNESCO de } \\
\text { Educación a Distancia, } \\
\text { UNED - Facultad de } \\
\text { Educación, C/ Juan del } \\
\text { Rosal, } 14,8040 \text { - Ciudad } \\
\text { Universitaria, Madrid-España }\end{array}$ & $\begin{array}{l}\text { htap://www.utpl.edu.edried/ } \\
\text { ried@edu.uned.es }\end{array}$ \\
\hline
\end{tabular}




\section{Asociación Internacional de Estudios en Comunicación Social. (Uruguay).} http://goo.gl/SWPsI

\begin{tabular}{l|r|}
\hline HOME ABOUT IAMCR SECTIONS \& WORKING GROUPS & CONFERENCE \\
Home > Open Access Journals & RESOURCES \\
\hline
\end{tabular}

CLARISE. Comunidad Latinoamericana Abierta Regional de Investigación Social y Educativa. (México, Argentina, Colombia, Uruguay y Costa Rica). http://goo.gl/VxlRI

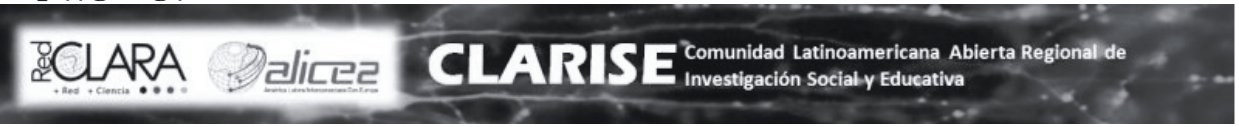

- RIED: Revista Iberoamericana de Educación a Distancia (ISSN:1138-2783)

http://www.utpl.edu.ec/ried/

Enlaces Educativos en español de la Universitat de València. (España). http://goo.gl/7dW3q

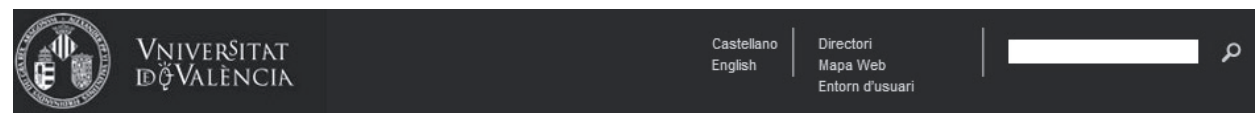

\section{ENLACES EDUCATIVOS EN ESPANOL}

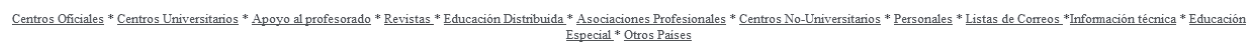

- $\underline{\text { RIED }}$. Revista Iberoamericana de Educación a Distancia 
Red Iberoamericana de Revistas de Comunicación y Cultura. (Argentina). http://goo.gl/3zd31

Red Iberoamericana de Revistas

de Comunicación y Cultura

\section{RIED. Revista Iberoamericana de Educación a Distancia}

Director: Dr. Lorenzo García Aretio (Cátedra UNESCO de Educación a Distancia - UNED, España).

Responsable editorial: José Barbosa Corbacho (Rector, Canciller UTPL, Loja, Ecuador).

Página web: http://www.utpl.edu.ec/ried/

Correo electrónico: ried@edu.uned.es

Teléfonos: $(+34) 913987218 /(+34) 913989340 /(+34) 913989061$

REDIAL. Red Europea de Información y Documentación sobre América Latina CEISAL. Consejo Europeo de Investigaciones Sociales de América Latina. (España) http://goo.gl/VNzox

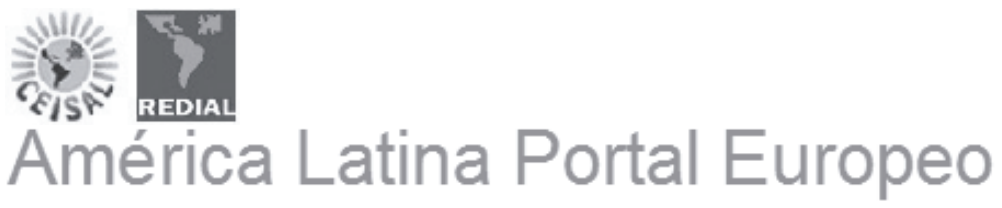

REDIAL Red Europea de Información y Documentación sobre América Latina CEISAL Consejo Europeo de Investigaciones Sociales de América Latina

\section{RIED. Revista Iberoamericana de Educación a Distancia}

Papel | Versión digital gratuita | Semestral | España

ISSN versión papel: $1138-2783$

Año de creación: 1998

Editor: Asociación Iberoamericana de Educación Superior a Distancia (AIESAD)

Página web del editor $\boldsymbol{t}^{\star}$

Dirección: Cátedra UNESCO de Educación a Distancia.

C/ Juan del Rosal, 14

28040 - Ciudad Universitaria.

Madrid (España) 


\section{BUSCADORES DE LITERATURA CIENTÍFICA}

Dulcinea. Derechos de copyright y de las condiciones de auto-archivo de revistas científicas españolas. (España). http://goo.gl/DibnF

\section{DULCINEA}

Derechos de copyright y las condiciones de auto-archivo de revistas científicas españolas

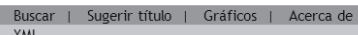

XML

RIED. Revista Iberoamericana de Educación a Distancia

Ficha básica Ficha completa

Editorial: UNED - UTPL

URL: http://www.utpl.edu.ec/ried/

Tipo de acceso: Gratuito

Mención específica de derechos: Sí

¿Permite el auto-archivo?: Si

Versión auto-archivo: Post-print (versión editorial)

Color Romeo: Azul

Enlaces a otros directorios: DIALNET - DOAJ - MIAR * RCS

Sherpa/Romeo. Publisher copyright policies \& self-archiving. (Reino Unido). http://goo.gl/UFVnm

\section{SHERPA/ROMEO}

opening access to research Home $\cdot$ Search $\cdot$ Journals . Publishers FAQ . Suggest $\cdot$ About

Journal: RIED (ISSN: 1138-2783, ESSN: 1390-3306) [started 1998]

RoMEO: This is a ROMEO blue journal

Listed in: DOAJ as an open access journal

Author's Pre-print: $\mathbf{X}$ author cannot archive pre-print (ie pre-refereeing)

Author's Post-print: $\mathbf{X}$ author cannot archive post-print (ie final draft post-refereeing)

Publisher's Version/PDF: $\checkmark$ author can archive publisher's version/PDF

General Conditions:

- Creative Commons Attribution Non-Commercial No-Derivatives

- Publisher's version/PDF must be used

- On personal website or Institutional Repository

Mandated OA: (Awaiting information)

Copyright: Polic

Updated: 20-Apr-2012 - Suqgest an update for this record

Link to this page: http://www. sherpa.ac ukJromeo/issn/1138-2783/

Published by: Asociación Iberoamericana de Educación Superior a Distancia (AIESAD) [Society Publisher] - Blue Policies in RoMEO

Other parties: 1. Universidad Nacional de Educación a Distancia (UNED) [ASsociate Oraanisation]- Sugqest to RoMEO

2. Universidad Técnica Particular de Loja (UTPL) [Associate Oraanisation]- Sugqest to RoMEO

Guidance: Please see the list of Publisher Categories in ROMEO for guidance on interpreting the priority of multiple publishers

\section{CATÁLOGOS DE BIBLIOTECAS}

Biblioteca de la Universidad Carlos III de Madrid. (España)

http://goo.gl/DkS6l

Universidad

Carlos III de Madrid

Biblioteca y mat

Source: RIED. Revista iberoamericana de educación a distancia [1138-2783] 
Biblioteca de la Universidad de Sevilla. (España). http://goo.gl/YMD6N

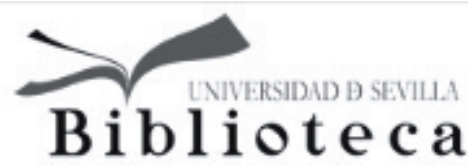

RIED : revista iberoamericana de educación a distancia. Madrid : UNED, 1998-

Acceso al Sumario

+ Ver fondos de la biblioteca

- Más detalles

DESCRIPC. Vol. 1, n.1(jun. 1998)-

URL http://dialnet.unirioja.es/servlet/revista?codigo=1295

ISSN 1138-2783

MATERIA Enseñanza a distancia -- Publicaciones periódicas.

CLASIF. ABREV. 37 (05)

CLASIFICAC. 378.018.43(05)

OTRA ENTIDAD Universidad Nacional de Educación a Distancia (España)

Biblioteca de la Universidad Autónoma de Madrid. (España).

http://goo.gl/56Ifi

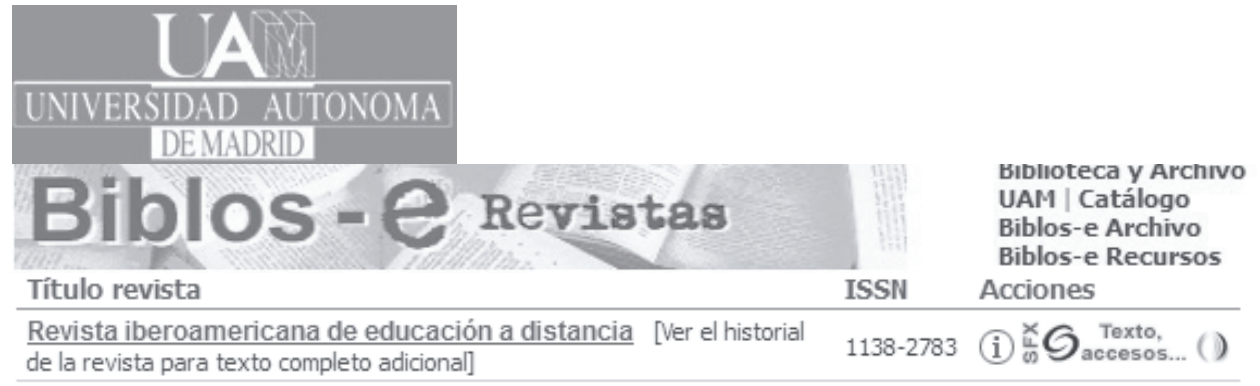




\section{Biblioteca de la Universidad de Málaga. (España).} http://goo.gl/rJV5l

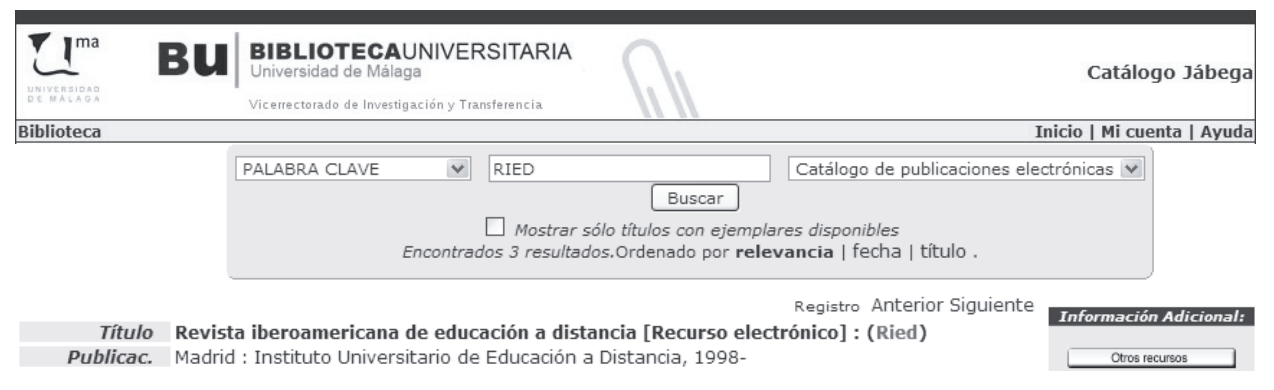

Biblioteca de la Universidad de Huelva. (España).

http://goo.gl/umHZK

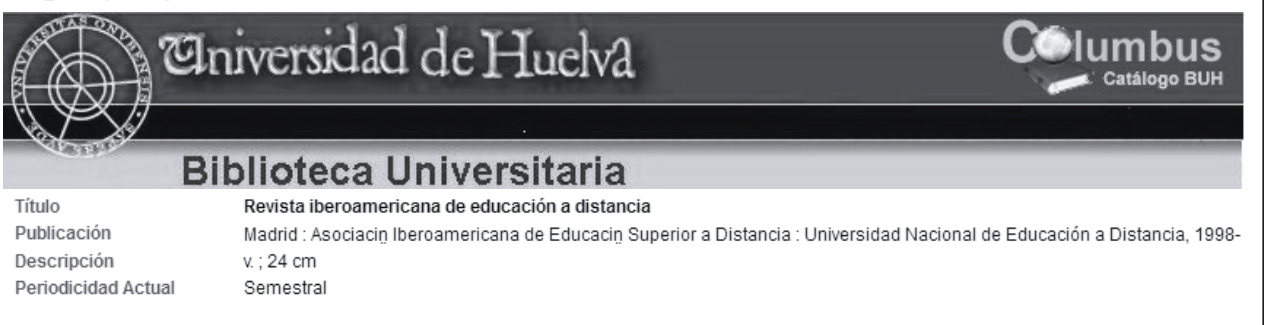

Biblioteca de la Universidad de Granada (España)

http://goo.gl/41CSe

Ugr Universidad de Granada

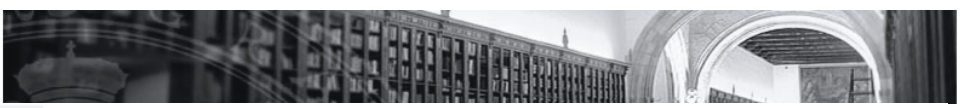

Título Clave

Revista iberoamericana de educación a distancia : (Ried)

Título

Revista iberoamericana de educación a distancia : (Ried)

Editor/Impresor

Loja (Ecuador) : Universidad Técnica Particular de Loja, 1998-

Por tanto, en el mes de noviembre de 2012 la situación de la RIED en las diferentes bases de datos y catálogos es la siguiente:

Bases de datos: BASE, CAPES (Coordenação de Aperfeiçoamento de Pessoal de Nível Superior), CEDAL (Instituto Latinoamericano de Comunicación Educativa (ILCE) de México), CIRC (Clasificación Integrada de Revistas Científicas), CREDIOEI (Centro de Recursos de la OEI), DIALNET (Alertas de Literatura Científica 
Hispana), IRESIE (Índice de Revistas de Educación Superior e Investigación Educativa -iisue-), HEDBIB (International Bibliographic Database on Higher Education), ISOC-CSIC/CINDOC-(Consejo Superior de Investigaciones Científicas de España), Psicodoc, REDINED.

Plataformas de evaluación de revistas: DICE (Difusión y Calidad Editorial de Revistas), IN-RECS (Índice de Impacto de Revistas Españolas de Ciencias Sociales), MIAR (Matriz para Evaluación de Revistas), QUALIS-CAPES, RESH (Revistas Españolas de Ciencias Sociales).

Directorios selectivos: LATINDEX (Publicaciones Científicas Seriadas de América, España y Portugal), ULRICH’S Periodicals (CSA).

Portales y repositorios especializados: Actualidad Iberoamericana, Asociación Internacional de Estudios en comunicación social, CLARISE (Comunidad Latinoamericana Abierta Regional de Investigación Social y Educativa), EDUC. $\mathrm{AR}$ (El portal educativo del estado argentino), Enlaces educativos en español de la Universitat de València , E-SPACIO-UNED (Repositorio institucional de la UNED), $360^{\circ}$ (Plataforma de revistas), Periódicos CAPES, Red Iberoamericana de Revistas de Comunicación y Cultura, REDIAL \& CEISAL, UNIVERSIA.

Buscadores de literatura científica: DOAJ (Directory of Open Access Journals), DULCINEA, GOOGLE ACADÉMICO, RECOLECTA (Recolector de ciencia abierta), SCIRIUS (For scientific information only), Serpa Romeo.

Catálogos de bibliotecas: Biblioteca de la UC3M, Biblioteca de la UNAM, Biblioteca de la Universidad Autónoma de Madrid, Biblioteca de la Universidad de Málaga, Biblioteca de la Universidad de Huelva, Biblioteca de la Universidad de Granada, BRITISH LIBRARY, BUZ, CARHUS Plus+, Catálogo de la Biblioteca de Educación (Ministerio de Educación, Cultura y Deporte), CCPP (Catálogo Colectivo de Publicaciones Periódicas Español), CIRBIC (Catálogo del CSIC), CCUC, CENDOC, CIDE, CISNE, COMPLUDOC, ICDL, INRP, IOE (Institute of Education. University of London), KINGS, MIGUEL DE CERVANTES, REBIUN, UBACAT, $U I B$, WORDLCAT, ZDB.

Asimismo, después del pertinente período de evaluación, la RIED ha sido aceptada para su inclusión en las bases de datos que detallamos a continuación. En estos momentos estamos finalizando el proceso de indización de nuestra revista en sus respectivos espacios Web: 
CLASE-Citas Latinoamericanas en Ciencias Sociales y Humanidades. Universidad Nacional Autónoma de México (UNAM). (México).

http://clase.unam.mx

Estamos a la espera de que incluyan la referencia a la RIED en esta base de datos.
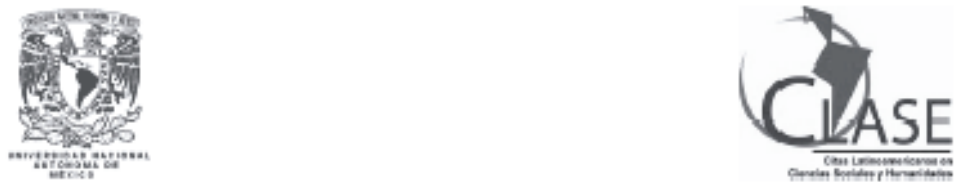

Direccion General de BIbllotecas

Subdireccion de Serviclos de Informacion Especlallzada

Bibilografla Latinoamericana. Hemeroteca Latinoamericana

\section{EBSCO Publishing. (Estados Unidos). \\ http://www.ebscohost.com/}

EBSCO nos ofrece la posibilidad de ser nosotros los encargados de subir los números completos y cada uno de los artículos de la RIED a su página Web. Para ello nos facilitan las claves de su FTP. Actualmente estamos realizando la subida de todo el contenido de la RIED.

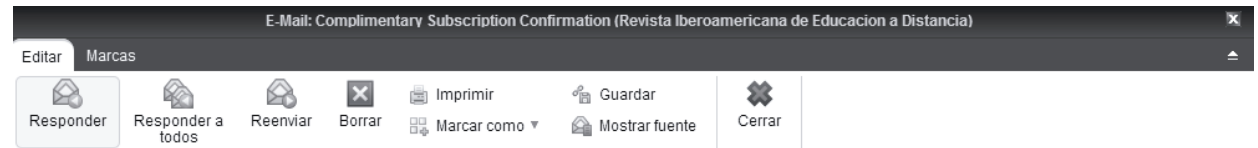

Complimentary Subscription Confirmation (Revista Iberoamericana de Educacion a Distancia)

De : $\quad$ "Julie Becker" <jbecker@ebscohost.com>

Para: Igaretio@edu.uned.es; ried@edu.uned.es

Recibido: 08/05/2012 18:34

Greetings,

We are very pleased to include the Revista Iberoamericana de Educacion a

Distancia in EBSCO's library products. I would like to arrange the

delivery of your title to EBSCO Publishing. The attached document

includes a brief description of delivery methods and format

requirements. Processing titles using PDF data is the best possible way

of presenting your publications in our library products.

If you can confirm that PDF data is available, I would be happy to

arrange your preferred method of delivery to EBSCO Publishing. If PDF

data is not available at this time, just let me know.

Thank you for your time and assistance with this request. Please feel

free to contact me with any questions.

Best regards,

Julie Becker, Serials Coordinator

EBSCO Publishing

Editorial Department

10 Estes Street

Ipswich, MA 01938

JSA

(978) $356-6500$

(800) $653-2726$

Fax (978) 356-7026

jbeckerlebscohost.com <mail to: jbeckerlebscohost.com〉 
Además, la RIED se encuentra en proceso de evaluación para ser indizada en:

- Scopus (Holanda): $\underline{\text { http://www.scopus.com }}$

- $\quad$ Redalyc (México): $\underline{\text { http://redalyc.uaemex.mx }}$

- e-Journals (Suiza): http://www.e-journals.org

- Hapi (Estados Unidos): http://hapi.ucla.edu

\section{CRITERIOS SOBRE LA CALIDAD DEL PROCESO EDITORIAL}

La calidad del proceso editorial es uno de los puntos principales que son tenidos en cuenta en las bases de datos nacionales e internacionales para que una revista sea incluida y valorada, por esta razón este es uno de los factores que se ha consolidado en el tiempo. No obstante, hay que destacar que durante este periodo de tiempo han existido algunos cambios relevantes dentro del proceso editorial para alcanzar una mejora sustancial. En este apartado se van a destacar aquellos cambios que se han producido siguiendo el siguiente orden:

- Volúmenes publicados y cambio en la periodicidad de la revista.

- Introducción de las normas de política editorial.

- OJS como proceso de gestión editorial.

\section{Volúmenes publicados y cambio en la periodicidad de la revista}

La RIED, Revista Iberoamericana de Educación a Distancia, ha afianzado, especialmente en los últimos años, los indicadores relevantes dentro de la calidad editorial. Así la RIED mantiene su edición de números con rigurosa periodicidad ajustándose permanentemente a las fechas de publicación.

No obstante, este último año se ha producido un cambio sustancial en el periodo de publicación de la revista, lo que ha conllevado diversas acciones encaminadas a conseguir mantener la periodicidad. Durante este último periodo que comprende esta memoria, se han publicado 3 volúmenes en lugar de dos, como se venía haciendo habitualmente. Este cambio responde a seguir cumpliendo con los criterios de calidad que nos solicitaban las bases de datos internacionales, ya que según los 
estudios era más valorado dentro de los estándares de calidad publicar en los meses de enero y julio, en lugar de los meses de diciembre y junio como se procedía hasta ese momento.

Por esta razón la RIED, para cumplir con la periodicidad, así han sido las fechas de publicación de los últimos cuatro números:

- 14.2 (diciembre de 2011)

- $\quad 15.1$ (enero de 2012)

- $\quad 15.2$ (julio de 2012)

- $\quad 16.1$ (enero de 2013)

A partir de estos datos se debe señalar que los trabajos recibidos durante este último año ascienden a un total de 98. Este número de artículos se distribuye de la siguiente manera:

- 27 artículos han sido publicados en los volúmenes 14.2, 15.1, y 15.2 obteniendo, por tanto, una evaluación positiva por parte de los miembros del Comité Científico y Evaluadores Externos.

- 15 trabajos más han sido valorados positivamente, 9 de ellos se han incorporado al volumen 16.1.

- 17 están aún en proceso de revisión externa por haberse recibido posteriormente.

- 39 artículos han sido rechazados y no serán publicados en la revista RIED.

Según estos datos, el porcentaje de los artículos aceptados, publicados y rechazados que se ha recibido en la secretaría de la RIED es el siguiente: 


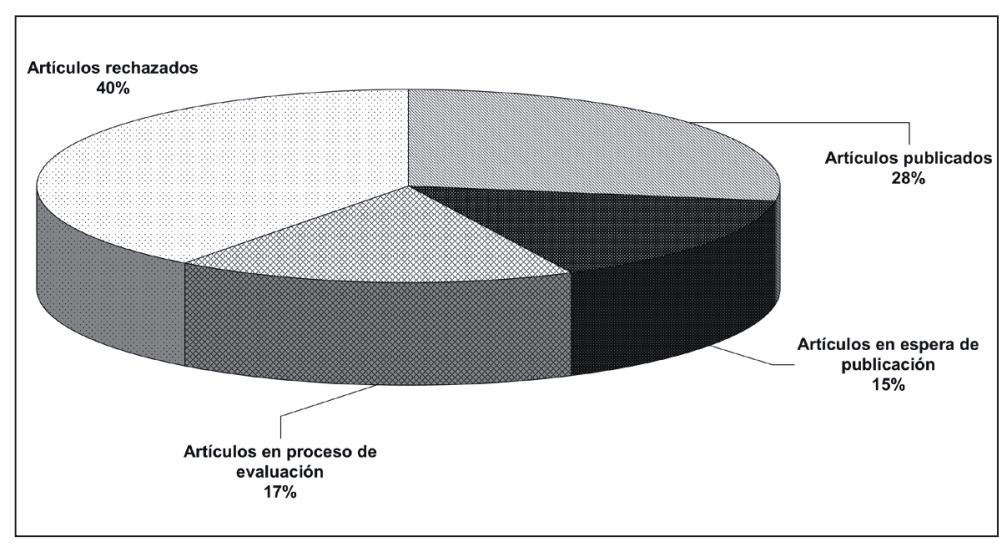

Gráfico 3. Porcentajes de artículos recibidos

Un aspecto que es importante destacar es la apertura y difusión de la RIED dentro de la comunidad científica, tal y como señalábamos en el apartado anterior. Una de las formas de conocer la difusión de nuestra revista es el análisis de los colaboradores que publican o desean publicar. Así, en la siguiente gráfica podremos observar los países de procedencia de los artículos que se han publicado en los dos últimos volúmenes que han sido editados en este último año:

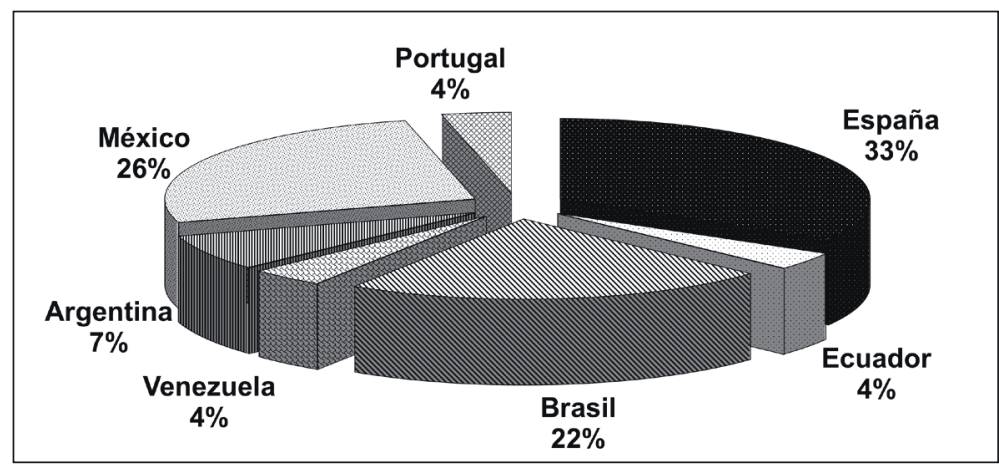

Gráfico 4. Porcentajes de países de procedencia de artículos publicados

\section{Introducción de las normas de política ética y de responsabilidad editorial}

Uno de los nuevos indicadores, que son requisitos para las más prestigiosas bases de datos de índole internacional, es la llamada política ética y de responsabilidad 
editorial. En estos momentos la RIED ya tiene este apartado que puede ser consultado a través de su página Web: http://www.utpl.edu.ec/ried/?q=es/node/89

Este apartado es un ejercicio de transparencia que presenta la revista como forma de cumplir con los estándares de calidad establecidos. Se destaca la política de privacidad donde se reconoce que se mantendrá y preservará en todos los casos y circunstancias el anonimato de los autores y el contenido de los artículos desde la recepción del manuscrito hasta su publicación.

Aunque también es importante subrayar que en este mismo apartado se trata de uno de los temas que ya hemos destacado como es el que los textos publicados en esta revista están sujetos a una licencia "Reconocimiento-No comercial 3.o" de Creative Commons.

\section{OJS (OPEN JOURNAL SYSTEMS) como proceso de gestión editorial}

OJS es un software de código abierto desarrollado por el Public Knowledge Project (PKP) de Canadá, organismo dedicado al desarrollo y aprovechamiento de nuevas tecnologías para la investigación académica y para el acceso universal a la producción científica.

Se trata de un sistema de administración y publicación de revistas científicas y académicas en Internet diseñado para mejorar la gestión de las publicaciones seriadas. Permite un manejo eficiente y unificado del proceso editorial y facilita el acceso al conocimiento producido por universidades y centros de investigación. En los últimos años, OJS se está consolidando como la principal herramienta para el acceso a texto completo de los documentos publicados en revistas científicas de cualquier parte del mundo.

La principal característica y ventaja de OJS consiste en que permite integrar y organizar todas las tareas de gestión y administración de una publicación electrónica en un mismo espacio virtual y con una misma herramienta. Esto significa que todo el proceso llevado a cabo para la publicación de cualquier revista científica -el envío de los artículos por parte de los autores, la comunicación con cualquier miembro de la comunidad de la revista, la gestión de los originales para su posterior evaluación, el proceso de revisión y corrección, etc.- se ejecuta, siempre y en todo momento, a través del sistema OJS. 
Otras de las características que podemos enumerar como ventajas de este sistema, son las siguientes:

1. El OJS es instalado y administrado localmente.

2. Cada editor configura los requerimientos, secciones, proceso de revisión, etc.

3. Los envíos de artículos y todo el proceso editorial son controlados en línea.

4. Soporta diferentes módulos para ofrecer distintas opciones de acceso abierto.

5. Cuenta con un sistema de indexación de documentos a través del texto completo y los metadatos.

6. Sus herramientas de lectura están basadas en opciones habilitadas por cada editor.

7. Maneja notificaciones por correo electrónico del avance que hay en el proceso editorial y permite a los usuarios registrados comentar los artículos publicados.

8. Contiene un completo sistema de ayuda.

El OJS maneja el proceso editorial de una publicación periódica mediante cinco pasos, los cuales pueden ser ejecutados por uno o más editores:

1. Cola de envíos: flujo de artículos enviados. Comienza por el editor de la revista.

2. Revisión del envío: el autor siempre estará enterado del estado de su artículo y su aceptación o denegación.

3. Edición del envío: los artículos son enviados a maquetación, diagramación, revisión de estilo y sintaxis.

4. Programación de ejemplares: los artículos son programados para ediciones presentes o futuras sin límite de tiempo.

5. Tabla de contenidos: los artículos son ordenados por el editor de acuerdo al número y volumen del ejemplar. 
OJS posee unos roles de usuario encargados de las diversas tareas en el proceso editorial, desde el ingreso de artículos hasta su publicación, todo esto siguiendo un proceso lógico de flujo de trabajo. De esta forma, cada perfil de usuario tiene asignado un nivel de administración específico, desde el perfil del lector hasta el de administrador general.

No obstante, como ya se comentó anteriormente, el gestor encargado de cada revista podrá configurar su espacio como considere oportuno, pudiendo desestimar la utilización de cualquiera de los roles o funciones disponibles en OJS. En este sentido el sistema ofrece múltiples herramientas y funcionalidades, pero será cada revista la que establezca de qué manera se organiza la gestión y administración de sus contenidos.

Son ya numerosas las revistas científicas en todo el mundo, y de la mayoría de los ámbitos de conocimiento, que están utilizando OJS para la publicación y gestión de sus publicaciones electrónicas. Presentamos a continuación algunos ejemplos de revistas dentro de los ámbitos de educación y universidad que se publican y gestionan a través de $O J S$ :

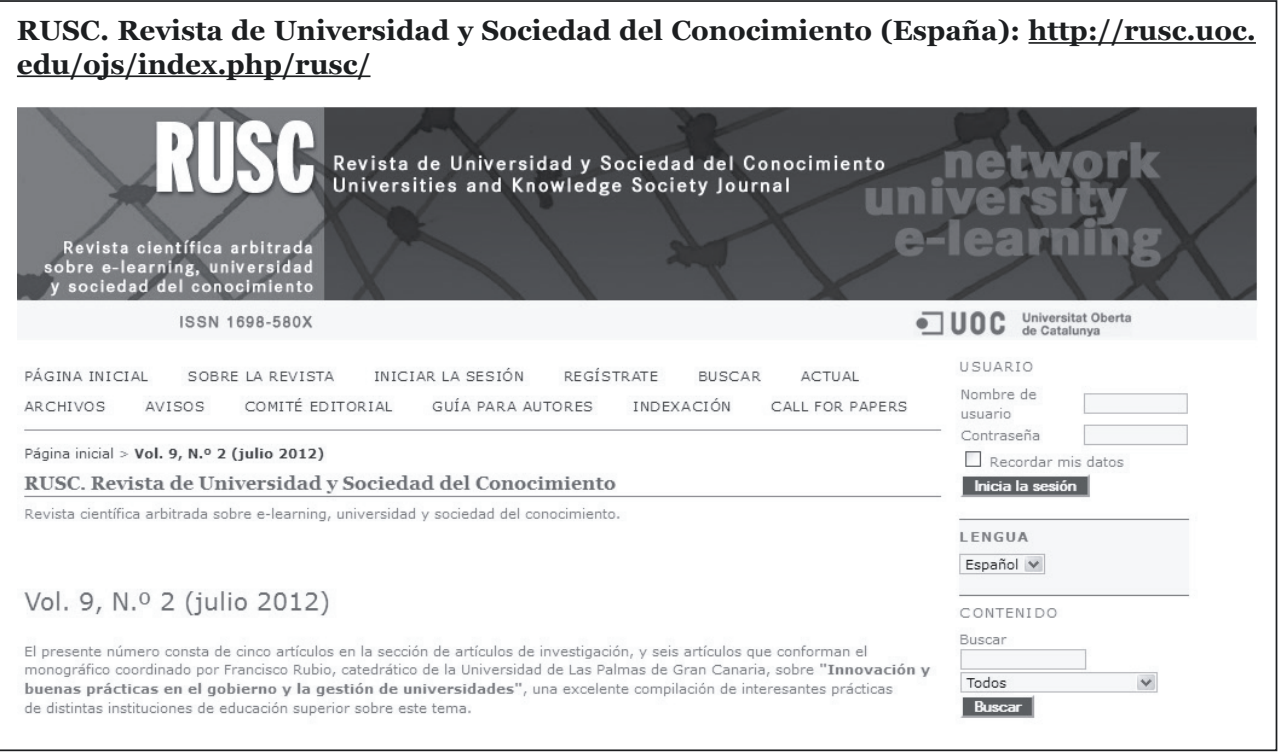




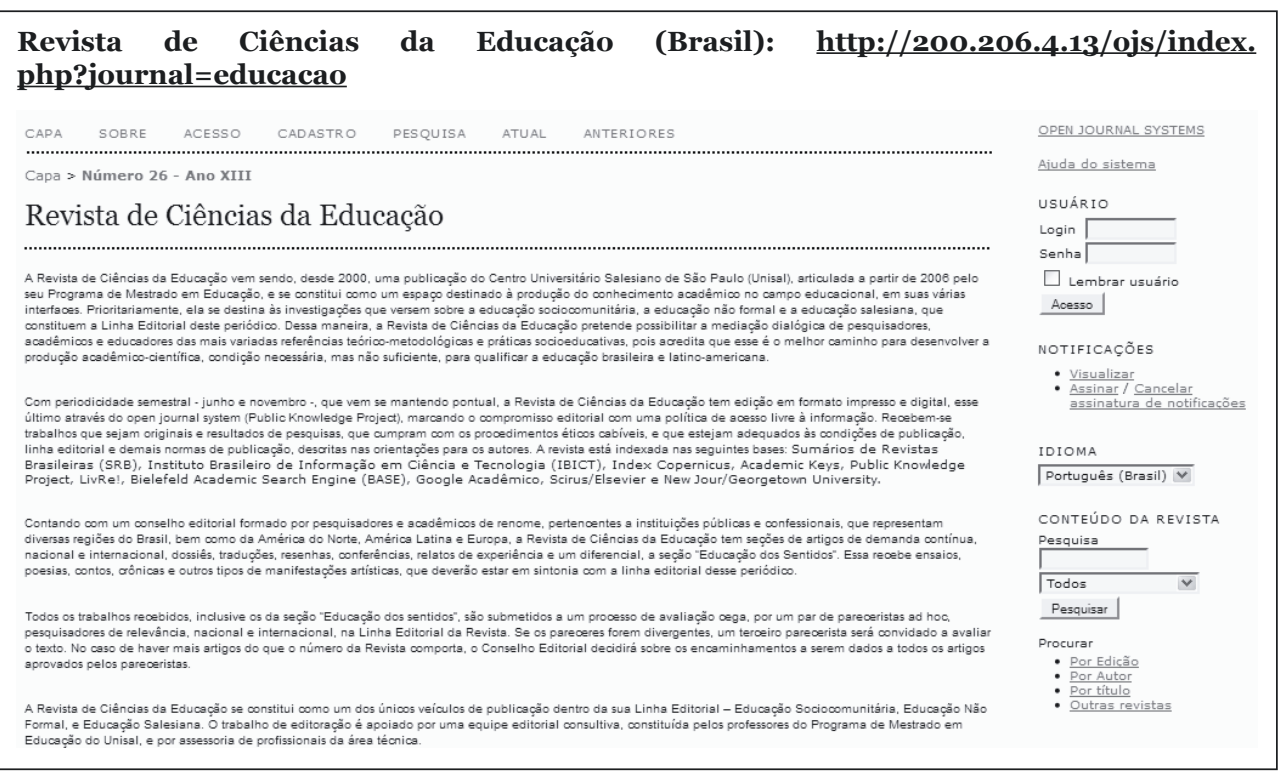

Revista Colombiana de Educación (Colombia): http://www.pedagogica.edu.co/revistas/ ojs/index.php/RCE

\section{R E V I S T A C O O M B I A A D E}

$$
\text { E D U C A C I Ó N }
$$

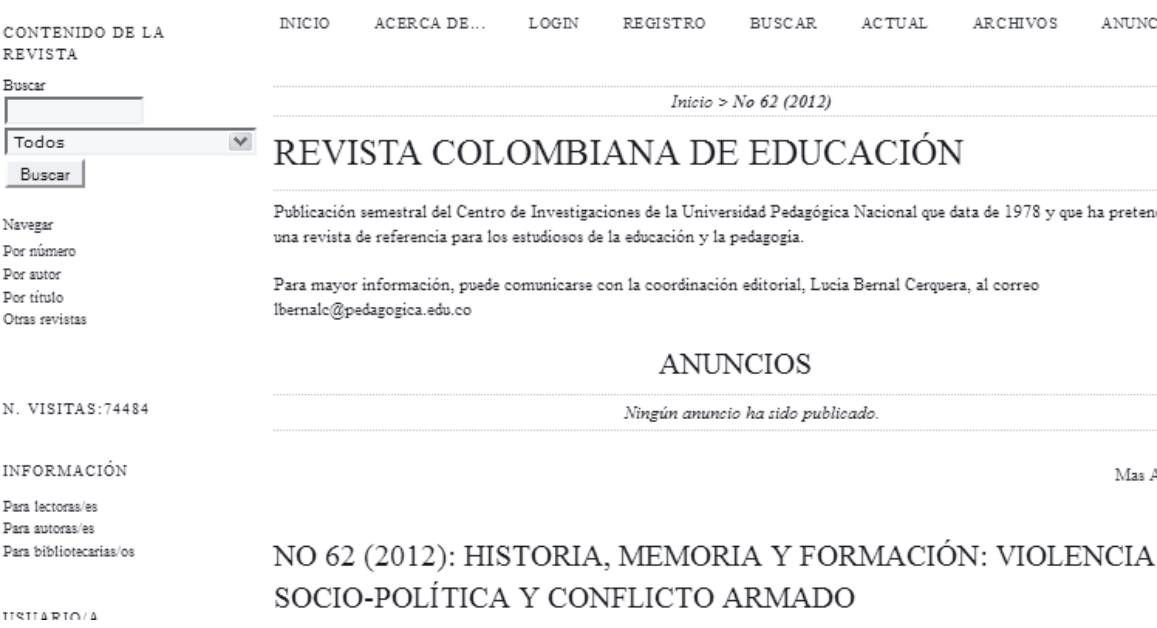


Revista Teoría de la Educación (España): http://campus.usal.es/ revistas trabajo/ index.php/revistatesi

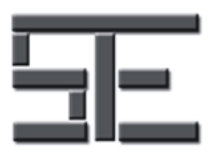

\section{Teoria de la Educación \\ Educación y Cultura en la Sociedad de la Información}

Teoría de la Educación. Educación y Cultura en la Sociedad de la Información

TESI es una revista fundada en 1998 cuyo campo de estudio es el impacto cultural del desarrollo de las Tecnologías de la Información y la Comunicación en los procesos de formación, el imaginario cultural, los discursos educativos, los escenarios de formación y las prácticas pedagógicas. Su director es Joaquín García Carrasco, catedrático de la Universidad de Salamanca. La revista tiene una periodicidad cuatrimestral (3 números al año), con posibilidad de editar números extraordinarios. La sede institucional de la revista es el Instituto Universitario de Ciencias de la Educación de la Universidad de Salamanca.

Puede consultar los números anteriores a diciembre de 2009 en este enlace.

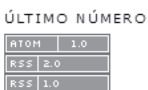

USUARIO

Nombre de usuario Contraseña $\square$ Recordar mis datos Login

CONTENIDO DE LA REVISTA Buscar

Buscar

Buscar Navegar

The International Review of Research in Open and Distance Learning (Canadá): http:// www.irrodl.org/index.php/irrodl/article/view/29

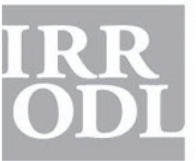

THE INTERNAIIONAL

REVIEW OF RESEARCH IN

OPEN AND DISTANCE LEARNING

A refereed e-journal to advance research, theory, and practice in open and distance learning worldwide.

HOME ABOUT REgister aRCHIVES ANNOUNCEMENTS RESOURCES SUBMISSions REVIEWERS IONFERENCES

fomes $\operatorname{Vol} 2, \mathrm{NO}_{1}(2001)$ > Garcia Aretio

The Technological Consolidation of UNED in Spain

Lorenzo Garcia Aretio

\section{Abstract}

This article discusses the role of the technologies that have been utilized to advance distance teaching and learning by the National Distance Education University (Universidad Nacional de Educación a Distancia - UNED) of Spain. Following a description of UNED's historical developmen and organizational structure, UNED sexperience with and video recordings, telephone, videoconferencing computer systems and computer-mediated communications are also described UNgD's pioneeri projects, including the virtual classroom, virtual campus and a program for the physically handicapped, are also detailed. Recent experiments include providing access to radio and television programs on the internet and adoption of WebCT. On the horizon for UNED are portals for cellular phones using wA.P technology and gearing up for multiple applications in accordance with Universal stobile Telecommunications Technology (UMTS)

Lorenzo García Aretio is a Doctor in Educational science, Professor of Education, and UNEsco Chair in Distance Education at the National Distance Education University (UNED) of Spain. He has also been Director of the University Institute of Distance Education at UNED. As a writer and editor, Lorenzo Garcia Aretio has published 15 books on distance education. He has also written more than 70 articles and chapters for various distance education journals and books.

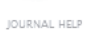

OPEN JOURNAL STSTEMS

ISSN: $1492-38_{31}$

SEARCH

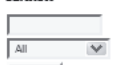

Search

BROWSE

: By testa

USER

Usmam

Passourd

$\square \mathrm{R}$.

$\log \ln$

CURRENT ISSUE

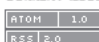

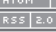

ARTICLE TOOIS 


\section{American Journal of Education (Estados Unidos): http://www.press.uchicago.edu/ ucp/journals/journal/aje.html}

\section{THE UNIVERSITY OF CHICAGO PRESS JOURNALS}

gOOKS | \{ JOURNALS \} DISTRIBUTION INFO \& SERVICES

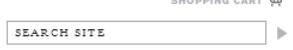

American Journal of Education

Sponsored by the Penn State College of Education

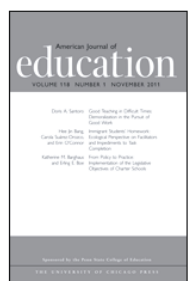

Frequency: 4 issues/year; ISSN: 0195-6744; E-ISSN: 1549-6511 Editors: Gerald LeTendre, Pennsylvania State University

The American Journal of Education seeks to bridge and integrate the intellectual, methodological, and substantive diversity of educational scholarship, and to encourage a vigorous dialogue between educational scholars and practitioners. AJE publishes research, theoretical statements, philosophical arguments, and critical syntheses of a

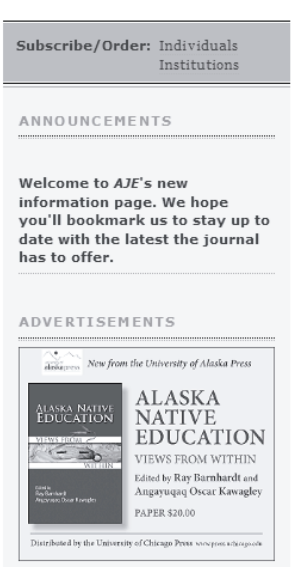

4 SHARE GY

Read Journal Online

Most Cited

La RIED ya cuenta con su propio espacio OJS completamente diseñado y configurado. Como se puede apreciar, los logos principales que figuran en el encabezado de todas las páginas de la RIED dentro del espacio OJS son el de la UNED, el de la UTPL, el de la AIESAD y, por supuesto, el de la RIED:

\section{La RIED en OJS: http://e-spacio.uned.es/revistasuned/index.php/ried}

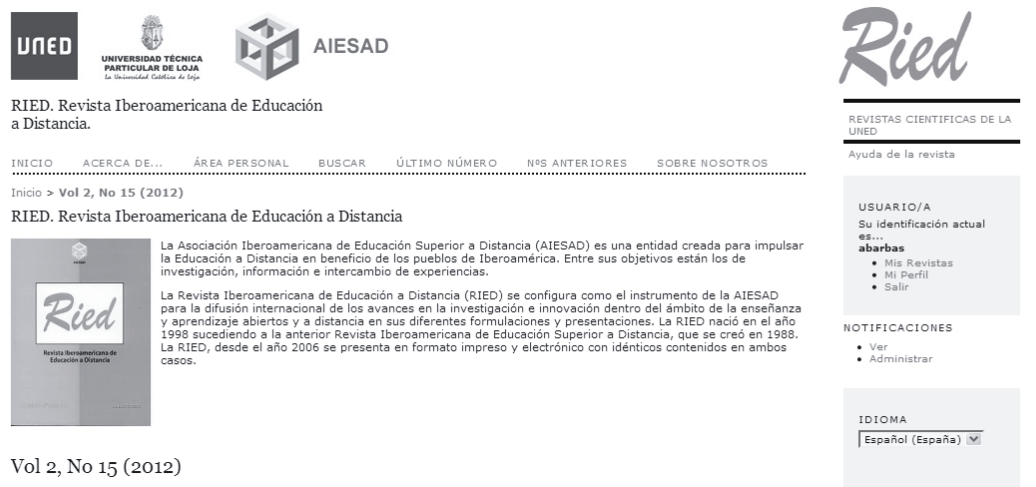


Por todos los motivos anteriormente expuestos se adoptó la decisión de que la $R I E D$ debía apostar decididamente por el alojamiento, la gestión y la difusión en $O J S$ y a través de $O J S$.

\section{CRITERIOS SOBRE LA CALIDAD CIENTÍFICA DE LA REVISTA}

A partir de este momento, tenemos que marcarnos como referencia otras líneas que hemos puesto en marcha durante este último año y que ya se están llevando a cabo.

- Contenido editorial: Para que la RIED sea incluida en las más prestigiosas bases de datos nacionales e internacionales es necesario que aporte investigaciones y estudios innovadores y de máxima calidad. Es vital que los trabajos que se publiquen tengan gran rigor científico y que sean originales. Esta es una labor fundamental que están realizando los miembros del Comité Científico y los Evaluadores Externos, pero que a su vez es una responsabilidad de la dirección de la revista.

- Internacionalidad de autores: Como se veía en el gráfico 4, en los dos últimos volúmenes ya se ha tenido en cuenta este aspecto, no obstante, si nos remitimos a los trabajos recibidos todavía son mayoritarios los que corresponden a autores españoles.

- Análisis de citas: Las bases de datos evalúan que las revistas sean citadas dentro de su campo. Esto es un sinónimo de la difusión de la revista, pero también de la calidad de los trabajos que se publican en ella. En este sentido se ha comenzado a trabajar en tres ejes fundamentales:

- La incorporación en cada uno de los artículos que aparecen en la Web de la RIED de la correspondiente referencia para que los artículos puedan ser citados con mayor facilidad.

- Este año se ha incluido en los índices de cada volumen todos los títulos de cada uno de los artículos en inglés.

- Otra de las medidas tomadas es la adquisición del E-ISSN para que puedan ser citados los artículos que están en la Web de la RIED. 
El análisis que se ha presentado en esta memoria de actividades sobre la evolución de la RIED ha explicado y demostrado todas las mejoras incorporadas. No obstante, también es fundamental tener en cuenta que la clave no es solo el avance de la revista sino que es imprescindible que los progresos se consoliden y se amplíe nuestra difusión a través de la inclusión de la RIED en más bases de datos nacionales e internacionales.

El presente informe ha sido elaborado por:

Lorenzo García Aretio

María García Pérez

Ángel Barbas Coslado 